\title{
AMI Communication Requirements to Implement Demand-Response: Applicability of Hybrid Spread Spectrum Wireless
}

MD Hadley

SL Clements

TE Carroll

September 2011

\section{Pacific Northwest}

NATIONAL LABORATORY

Proudly Operated by Battelle Since 1965 



\title{
DISCLAIMER
}

This report was prepared as an account of work sponsored by an agency of the United States Government. Neither the United States Government nor any agency thereof, nor Battelle Memorial Institute, nor any of their employees, makes any warranty, express or implied, or assumes any legal liability or responsibility for the accuracy, completeness, or usefulness of any information, apparatus, product, or process disclosed, or represents that its use would not infringe privately owned rights. Reference herein to any specific commercial product, process, or service by trade name, trademark, manufacturer, or otherwise does not necessarily constitute or imply its endorsement, recommendation, or favoring by the United States Government or any agency thereof, or Battelle Memorial Institute. The views and opinions of authors expressed herein do not necessarily state or reflect those of the United States Government or any agency thereof.

\author{
PACIFIC NORTHWEST NATIONAL LABORATORY \\ operated by \\ BATTELLE \\ for the \\ UNITED STATES DEPARTMENT OF ENERGY \\ under Contract DE-AC05-76RL01830 \\ Printed in the United States of America \\ Available to DOE and DOE contractors from the \\ Office of Scientific and Technical Information, \\ P.O. Box 62, Oak Ridge, TN 37831-0062; \\ ph: (865) 576-8401 \\ fax: (865) $576-5728$ \\ email: reports@adonis.osti.gov \\ Available to the public from the National Technical Information Service, \\ U.S. Department of Commerce, 5285 Port Royal Rd., Springfield, VA 22161 \\ ph: (800) $553-6847$
fax: $(703) 605-6900$ \\ email: orders@ntis.fedworld.gov \\ online ordering: http://www.ntis.gov/ordering.htm
}




\title{
AMI Communication Requirements to Implement Demand-Response: Applicability of Hybrid Spread Spectrum Wireless
}

\author{
MD Hadley \\ SL Clements \\ TE Carroll
}

September 2011

Prepared for the U.S. Department of Energy

under Contract DE-AC05-76RL01830

Pacific Northwest National Laboratory

Richland, Washington 99352 


\section{Contents}

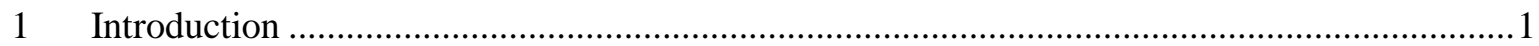

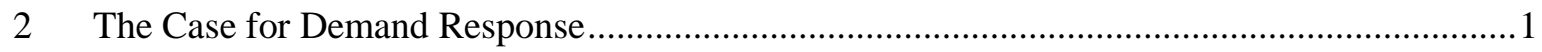

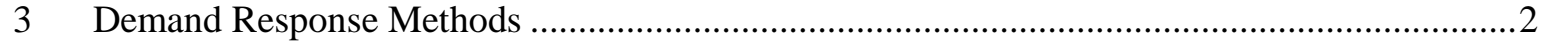

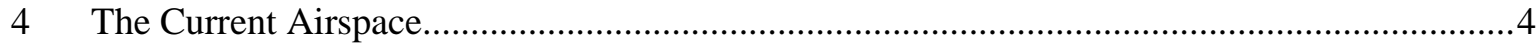

4.1 Method 1 - Reduce Density of Meters per Tower ..........................................................

4.2 Method 2 - Reduce Size of Real-Time Pricing Signals .................................................. 5

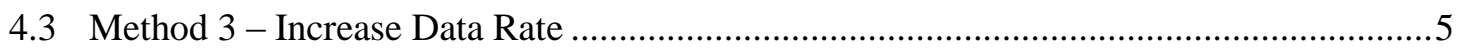

5 Hybrid Spread Spectrum (HSS) Wireless Telecommunications ...........................................6

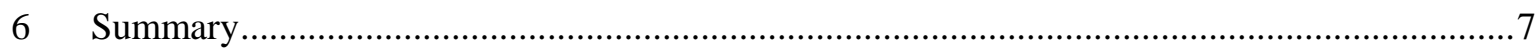




\section{Introduction}

While holistically defining the smart grid is a challenge, one area of interest is demand-response. In 2009, the Department of Energy announced over \$4 billion in grant and project funding for the Smart Grid. A significant amount of this funding was allotted to utilities for cost sharing projects to deploy Smart Grid technologies, many of whom have deployed and are deploying advanced metering infrastructure (AMI). AMI is an enabler to increase the efficiency of utilities and the bulk power grid.

The bulk electrical system is unique in that it produces electricity as it is consumed. Most other industries have a delay between generation and consumption. This aspect of the power grid means that there must be enough generation capacity to meet the highest demand whereas other industries could over produce during off-peak times. This requires significant investment in generation capacity to cover the few days a year of peak consumption. Since bulk electrical storage doesn't yet exist at scale another way to curb the need for new peak period generation is through demand-response; that is to incentivize consumers (demand) to curtail (respond) electrical usage during peak periods.

Of the various methods proposed for enabling demand-response, this paper will focus on the communication requirements for creating an energy market using transactional controls. More specifically, the paper will focus on the communication requirements needed to send the peak period notices and receive the response back from the consumers.

\section{The Case for Demand Response}

Real-time pricing, also known as transactive control, and feedback signals are elements necessary to implement demand response. One might ask why implement demand response at all? Isn't the current practice of load shedding sufficient? Consider the following scenario. Suppose that a utility decides that rather than add generation it would like to defer load. The situation could be:

a) for an emergency caused by sudden loss of generation, for example, or

b) it could simply be a matter of dispatching convenience,

c) or possibly a matter of economics where the utility will have to buy electricity on the spot market at a higher rate.

A question to answer would be this: Is there a qualitative difference between these load shedding situations?

In some implementations, there is a perceived difference between the situations. The utility, short on capacity, will simply open a circuit breaker and load will be shed. The decision is unilateral and one to which all of us can relate. This is what many in California experienced during the rolling blackouts in 2000 and 2001. There was not enough available generation to cover the demand and the partial deregulation at the time didn't allow retail electrical rate adjustments. Thus, even though there was a dire need to conserve energy, there was no incentive for the consumer to do so. Perhaps this approach characterizes the old power grid. In a Smart Grid implementation, there is no difference in the essence 
of the situations. A load reduction is desired in all cases; it is just that the emergency case is more urgent than the others. In each case, an incentive signal could be communicated from the local utility to the customer in near real-time. The customer could make a rational decision about whether to defer his or her electric usage or ignore the incentive signal and pay at an increased rate $f$. The situation with the sudden loss of generation could lead to a more urgent incentive to defer energy use.

\section{Demand Response Methods}

Two methods are currently available to implement demand-response technologies for these scenarios. The first is to deploy autonomous systems that require no interaction from consumers or utilities. These systems automatically sense changing conditions of the power grid and adjust accordingly. The second method is to communicate an incentive signal through the utility to each consumer. Both of the approaches are briefly discussed below.

One example of an autonomous system that can respond to power grid conditions by altering energy use is the Grid Friendly Appliance ${ }^{\mathrm{TM}}$ (GFA) Controller developed at Pacific Northwest National Laboratory. The GFA Controller senses grid conditions by monitoring frequency. A disturbance of the 60 $\mathrm{Hz}$ frequency indicates an imbalance between supply and demand. When the local grid frequency drops below $59.95 \mathrm{~Hz}$, the GFA Controller prompts the appliance to shed load. For example, with a clothes dryer, the heating element will be turned off while the drum is still allowed to spin. The result is $90 \%$ reduction in load and minimal adverse impact upon the consumer. After grid conditions return to normal, the GFA Controller prompts the appliance to return to normal operational mode in a manner that eases power restoration ${ }^{1}$.

Several benefits exist with this approach. First, no external communication is required to add intelligence to appliances. Second, with a deployment density of only 20\%, GFA Controller enabled appliances can positively impact the grid. Third, issues such as privacy and energy market manipulation are no longer of concern. The drawback is that this approach will only help provide grid stability to an emergency situation presented above.

The second approach is to communicate real-time price information to consumers thereby providing sufficient information to modify energy usage. Two signals are used in most implementations. The first is the Transactive Incentive Signal (TIS). The TIS is sent by the utility to each consumer. The TIS contains expected energy prices for upcoming periods of time. The second is the Transactive Feedback Signal (TFS) The TFS is sent from the consumer to the utility and contains information regarding expected energy use at various prices. The TIS and TFS together comprise an energy market where real-time price information is communicated to consumers. Real-time pricing can be used to satisfy all situations presented earlier.

\footnotetext{
${ }^{1}$ Grid Friendly Appliance ${ }^{\mathrm{TM}}$ Controller - Available Technologies - PNNL Retrieved September 30, 2011 from http://availabletechnologies.pnnl.gov/technology.asp?id=61
} 
As of the date of this paper, no standard exists that defines real-time price communication at the consumer level. The descriptions used in this paper are modeled after existing and proposed systems but do not define an exact implementation today. The purpose of this approach is two-fold. The first objective is to illustrate potential shortcomings that could occur if existing AMI environments are used for real-time pricing. The second objective is to show that the expected data rate and density capabilities of a Hybrid Spread Spectrum (HSS) environment may be better suited for demand response communications than existing solutions. The following XML example is based upon the data exchange format used by the Pacific Northwest Smart Grid Demonstration Project ${ }^{2}$. The data exchanged in this reference project provides 72 hours of 5-minute market data to the utility. The consumer-focused example uses 15 minute market data with a one-hour look ahead.

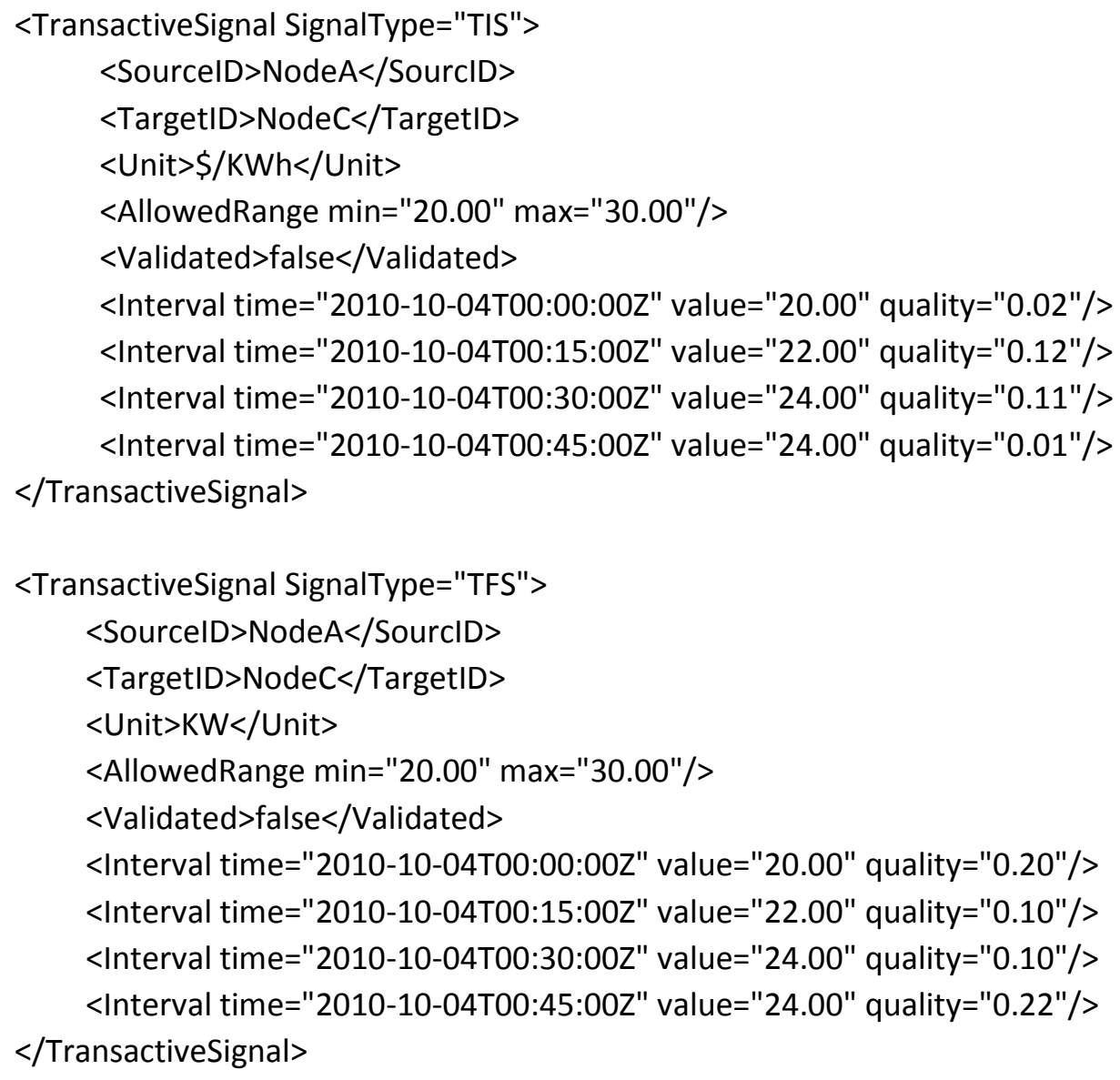

However, not everyone is in agreement that real-time pricing will lead to greater grid stability. A recent research paper by the Massachusetts Institute of Technology, "Volatility of Power Grids under Real-Time Pricing ${ }^{3}$," identifies potential problems with the use of pricing signals to facilitate demand response. In their paper, Roozbehani, Dahleh, and Mitter used mathematical modeling, theoretical analysis, and

\footnotetext{
${ }^{2}$ Pacific Northwest Smart Grid Demonstration Project, Implementation Design of Transactive Node

${ }^{3}$ Roozbehani, M. et. al.(2011, June 7) Volatility of Power Grids under Real-Time Pricing. Massachusetts Institute of Technology Retrieved September 29, 2011 from http://web.mit.edu/dahleh/www/pubs/Pubs.Roozbehani.Cornell.2011.pdf
} 
numerical simulations to study the effects of real-time pricing on the stability and volatility of electricity markets, and showed that exposing the retail consumers to the real-time wholesale market prices could create an unstable closed loop feedback system. The intent of this paper is not to debate this issue. However, as Smart Grid technologies are deployed, careful attention to the expected and real outcomes of transactive control and feedback messages must be measured.

\section{The Current Airspace}

Wireless technologies and power line carrier are two common methods to communicate TCS and TFS information to the consumer. We will focus solely on wireless technologies for this paper. What are the requirements for bandwidth and meter density? Should all customer meters be simultaneously accessible over the air waves? Should broadcast addressing be allowed to quickly communicate urgent requests to all meters? To address these questions, a representative AMI environment utilizing a 900 $\mathrm{MHz}$ network is described below.

\begin{tabular}{|l|l|}
\hline Attribute & Description \\
\hline Density of meters per tower & 50,000 \\
\hline Gross data rate for meter communication & $64 \mathrm{kbit} / \mathrm{s}$ \\
\hline Available data rate for real-time pricing & $51.2 \mathrm{kbit} / \mathrm{s}$ \\
\hline $\begin{array}{l}\text { Percent of data rate for processing and } \\
\text { communication timing }\end{array}$ & $25.6 \mathrm{kbit} / \mathrm{s}$ \\
\hline Effective data rate & $25.6 \mathrm{kbit} / \mathrm{s}$ \\
\hline TIS message size in above example & 533 bytes \\
\hline Frequency of TIS & Broadcast once every 15 minutes. \\
\hline TFS message size in above example & 533 bytes \\
\hline Frequency of TFS & 6 messages per second \\
\hline TIS responses per second & 1.8 \\
\hline Seconds for 50,000 TFS & 8,333 \\
\hline Minutes for 50,000 TFS & 139 \\
\hline Hours for 50,000 TFS & 2.31472 \\
\hline Conclusion & 15 minute market not possible with representative \\
& $\begin{array}{l}\text { AMI environment and XML-based TIS/TFS } \\
\text { communication. }\end{array}$ \\
\hline
\end{tabular}

What can be done to modify existing automated meter reading environments to enable real-time pricing for consumers? Three potential methods are identified below. Each method is explored in isolation, although combining methods will provide greater performance improvements. Methods include:

- reduce the density of meters per tower,

- reduce the size of the TIS/TFS messages, or

- increase the available data rate. 


\subsection{Method 1 - Reduce Density of Meters per Tower}

How many towers must be used to enable 15 minute response from all meters with the sample TIS/TFS? A constant rate of 3,333 meters per minutes $(50,000 / 15)$ will meet this requirement. The representative meter reading environment only supports 360 meters per minute $(60 * 6)$. A total of 9 towers $(3,333 / 360)$ will be needed.

\begin{tabular}{|l|l|}
\hline Attribute & Description \\
\hline Meters per minute & 3,333 \\
\hline TFS Reads per minute & 360 \\
\hline Towers needed to send 50,000 TFS in 15 minutes & 9 \\
\hline Conclusion & $\begin{array}{l}\text { Increasing the number of towers from 1 to 9 will } \\
\text { provide adequate meter density to enable 15 minute } \\
\text { real-time price forecast signals at current } \\
\text { communication rates. }\end{array}$ \\
\hline
\end{tabular}

\subsection{Method 2 - Reduce Size of Real-Time Pricing Signals}

Using a protocol format patterned after SCADA protocols such as DNP3 or Modbus will significantly reduce the message size. For example, assume the XML sample above can be defined as a protocol with fixed field lengths in a total of 64 bytes. The following table utilizes a single communication tower and the original $8 \mathrm{KBps}$ data rate. It may also be possible to use compression on the XML messages to reduce the size of the transmitted message.

\begin{tabular}{|l|l|}
\hline Attribute & Description \\
\hline TIS protocol format message size & 64 bytes \\
\hline TFS protocol format message size & 64 bytes \\
\hline Available data rate & 3,200 bytes per second \\
\hline Meters & 50,000 \\
\hline TFS responses per second & 50 \\
\hline Seconds for $\mathbf{5 0 , 0 0 0}$ responses & 1,000 \\
\hline Minutes for 50,000 responses & 16.66667 \\
\hline
\end{tabular}

While the 64-byte and our assumptions do not result in all TFS being communicated in 15 minutes, the total amount of time is decreased by over 2 hours. A message size of 57 bytes will provide the desired 15-minute response time.

\subsection{Method 3 - Increase Data Rate}

Another method to consider is increasing the data rate of the automated meter reading environment to meet the proposed 15 minute requirement. The same assumptions from before regarding percentage of communication for business systems and overhead for processing/timing of communication will be used in the calculations. To achieve 50,000 TFS in 15 minutes, 56 TFS messages must be sent every second 
$\left(50,000 /\left(15^{*} 60\right)\right)$. The following table shows the corresponding volume of data for these 56 messages in 30,000 bytes per second.

\begin{tabular}{|l|l|}
\hline Attribute & Description \\
\hline Meters per second & 56 \\
\hline Data rate for TFS & 30,000 bytes per second \\
\hline $\begin{array}{l}\text { Data rate for processing and timing of } \\
\text { communication }\end{array}$ & 30,000 bytes per second \\
\hline Data rate for business functions (add 20\%) & 15,000 bytes per second \\
\hline Required data rate for entire system & 75,000 bytes per second \\
\hline
\end{tabular}

Significant increases in data rate are required to meet the demands of the sample XML-based transactive forecast signal.

Existing AMI infrastructure may not be adequate to support the 15 minute markets proposed for realtime pricing. Emerging technologies need to ensure the ability to meet this requirement. Hybrid-Spread Spectrum wireless is one of these technologies.

\section{Hybrid Spread Spectrum (HSS) Wireless Telecommunications}

What is Hybrid Spread Spectrum? Hybrid-Spread Spectrum (HSS) combines two or more spread spectrum techniques for transmitting radio signals. Spread spectrum techniques spread information over a bandwidth that is larger than the inverse of the data rate. These techniques are used for a variety of reasons, including secure communications, resiliency against natural interference and jamming, preventing interception and detection, and limiting power flux density. The two most common techniques of spread spectrum are DSSS and FHSS. Direct-Sequence Spread Spectrum (DSSS) spreads the signal by multiplying the transmit signal by a second signal that has a very large bandwidth. The bandwidth of the resultant signal is approximately the same as the bandwidth of the wideband spread signal. Frequency-Hopping Spread Spectrum (FHSS) changes the carrier frequency of a narrowband so that transmission is done in one frequency band only for a short while. The resultant bandwidth is equal to the bandwidth over which the carrier frequency is hopped. Why combine DSSS and FHSS into a hybrid system? DSSS alone is useful for ameliorating the effects of multipathing, a phenomenon in which a transmission reaches the receiving antenna by two or more paths, but is neither spectrally efficient nor able to support the diverse types of links demanded by Smart Grid applications. Combining frequency hopping with direct sequences adds robustness, security, and good multiple-access properties for improved data transmission reliability and security, and, more importantly, greater capacity of simultaneous transmissions.

The anticipated HSS technology provides low-cost, high-integrity communications in support of utility operations across a range of applications, including sensor and control telemetry; remote metering; substation, switchyard, and relay monitoring; alarm transmission, and emergency communication. In the case of advanced metering, HSS is anticipated to offer both increased data rates and more simultaneous 
connections with the tower base station when compared to the typical meter infrastructure deployed today. Future work will test the HSS system for data rate and simultaneous connections per tower base station. The results will validate the applicability of the HSS wireless network as a suitable solution to provide real-time pricing to electric customers.

\section{Summary}

AMI is currently where SCADA was 15 to 20 years ago. Systems were designed to meet current, not future needs. AMI systems are currently in their infancy. Many changes will occur in the coming years including increased bandwidth and increased density of meters to radio base station. Priority of service and quality of service guarantees will be required to ensure real-time pricing signals are delivered as required for Smart Grid applications if a shared communication media is utilized. The ability of current AMI environments to enable real time pricing is limited. HSS is one potential technology to enable real-time pricing demand-response environments. However, two attributes must be present. First, the number of meters that can simultaneously connect to a tower and communicate over the RF spectrum must be significantly greater than current AMI deployments. Second, the available data rate must be sufficiently greater than current AMI systems. Performance testing of the HSS solution will be conducted by researchers at Pacific Northwest National Laboratory in fiscal year 2012. The sample TIS and TFS signals described in this paper will be used to measure the ability of HSS to support real-time pricing signals. A subsequent report will summarize those findings. 


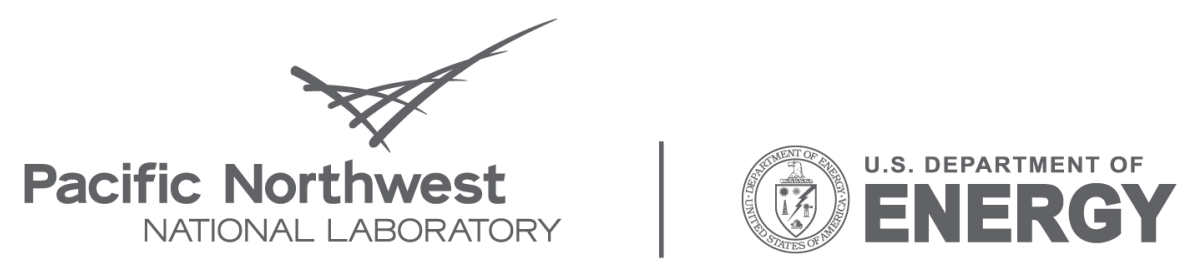

Proudly Operated by Battelle Since 1965

902 Battelle Boulevard

P.O. Box 999

Richland, WA 99352

1-888-375-PNNL (7665)

www.pnl.gov 